\title{
Inhibition d'une protéase coagulante fongique dans le lait cru
}

\author{
par \\ C. ALAIS*, P. DUCROO** et R. DELECOURT ${ }^{* *}$
}

\section{I. - INTRODUCTION}

Les enzymes coagulants employés pour le caillage du lait de fromagerie appartiennent au groupe des peptides-peptidohydrolases $(E C .3 .4 .4 . x)$ plus communément dénommés " protéases ». L'enzyme traditionnel dans de nombreuses contrées est la chymosine ou présure ; avec sa voisine, la pepsine, également utilisée pour la coagulation du lait, elle forme le sous-groupe des protéases acides, ( $\mathrm{pH}$ optimum de la protéolyse voisin respectivement de 4,0 et de 2,0). Les protéases coagulantes d'origine fongique, qui se répandent actuellement dans l'industrie sont des protéases moins acides $(\mathrm{pH}$ optimum d'activité sur la caséine supérieur à 5,0); certaines sont plutôt des protéases neutres, se rapprochant des protéases de la digestion intestinale.

Depuis les travaux de Laskowski $(1951,1952)$ on connaît la présence d'inhibiteurs de la trypsine dans le sérum sanguin humain et le colostrum. Ensuite, de nombreux travaux ont révélé que les inhibiteurs de trypsine étaient très répandus, aussi bien dans le règne végétal et chez les micro-organismes que dans le règne animal. De plus, on a montré l'existence d'inhibiteurs protéiniques naturels d'autres protéases animales : chymotrypsine, plasmine, thrombine, callicréine, etc., et de protéases végétales : papaïne, ficine, etc. Certains de ces inhibiteurs présentent une étroite spécificité ; d'autres sont capables d'inhiber des protéases variées. Il s'agit donc d'un phénomène très général (Fossum, 1970 ; Vogel et al., 1968).

Le colostrum contient des inhibiteurs de trypsine. La capacité d'inhibition de cet enzyme est différente d'une espèce à l'autre. Le colostrum de truie a une forte activité inhibitrice, celui de vache, une activité moyenne et le colostrum humain, une activité faible.

\footnotetext{
* Service de Biochimie appliquée, Université de Nancy I - 54037 Nancy.

** Service de Recherches, Société Rapidase - 59113 Seclin.
} 
Une substance inhibitrice cristallisée a été isolée des colostrums bovin et porcin ; mais il doit en exister bien d'autres, qui se distinguent de ceux du sang par leur mobilité électrophorétique (Fossum, 1970). Il est probable aussi que le lait, produit après la phase colostrale, contienne encore des inhibiteurs, mais en plus faible concentration.

En ce qui concerne la chymosine, les effets d'inhibition de ce type sont connus depuis longtemps mais ont été peu étudiés. Il y a plus de 70 ans, Briot (1900) montrait l'existence d'une " antiprésure " dans le sérum sanguin des animaux. Tsugo et Yamauchi (1953) ont isolé du sérum sanguin de divers mammifères un inhibiteur protéinique de la présure inactivé à $65-70^{\circ} \mathrm{C}$. Récemment, Singh et Ganguli (1973) ont observé la présence, dans le sérum sanguin bovin d'un inhibiteur de la chymosine distinct des inhibiteurs de la trypsine; il précipite avec les immuno-globulines et il ne semble pas affecter la réaction protéolytique proprement dite. Porcher (1930, 1931) a étudié l'empêchement de la coagulation du lait par la présure, exercé par une globuline du lait de vache ; ses conclusions sont en faveur de l'hypothèse du colloïde protecteur. C'est également la conclusion de Puhan $(1966,1967)$ qui a montré l'action de ralentissement de la coagulation par les globulines du colostrum ajoutées au lait ; il pense que les globulines agissent exclusivement sur le substrat (la caséine native) et n'ont aucune influence sur l'enzyme. Cependant, sur les 7 enzymes microbiens essayés par Puhan, 3 ne sont pas sensibles à l'inhibition dans le lait cru.

Des observations nouvelles sur la non proportionalité du temps de coagulation du lait de vache et de la dilution de l'enzyme, dans le cas de protéases coagulantes fongiques produites par des souches de Mucor miehei, permettent de penser que ce lait renferme un ou des inhibiteurs de telles protéases, qui sont inactifs sur la présure.

Dans les expériences qui font l'objet de cette première communication, nous avons cherché à préciser les caractéristiques essentielles de l'inhibition d'une protéase industrielle sensible au lait cru.

\section{II. - MATERIEL ET METHODES}

\section{1) Enzymes}

L'enzyme coagulant fongique est produit par Mucor miehei ; il est dénommé "Fromase » par le fabricant (Société Rapidase, Seclin). Quatre lots différents ont été essayés ; ce sont des préparations en poudre dont l'activité coagulante est de $1 / 100000$ ou 1/150 000 (unités Soxlhet). Pour les épreuves de comparaison, nous avons utilisé une présure animale en poudre du commerce. 


\section{2) Epreuve de coagulation}

Des solutions d'enzymes de concentration variée ont été préparées par dilution d'une solution mère dans l'eau distillée, de manière à obtenir des temps de coagulation allant de 3 à $50 \mathrm{mn}$; on ne peut mesurer avec assez de précision des temps de coagulation plus longs.

Le temps de coagulation est déterminé au bain-marie à $32^{\circ} \mathrm{C}$, dans des tubes à essai contenant $10 \mathrm{ml}$ de lait ; c'est le temps s'écoulant entre le moment de l'addition de l'enzyme $(0,5 \mathrm{ml})$ et l'apparition de flocons sur la paroi du tube incliné subissant un lent mouvement de rotation.

\section{3) Lait}

Du lait cru de plusieurs origines a été utilisé ; lait de ferme et lait de mélange de deux usines. Pour l'étude de l'effet du traitement thermique, de petites quantités de lait sont rapidement chauffées à la température choisie, maintenues à cette température durant $20 \mathrm{~s}$ ou $5 \mathrm{mn}$, puis rapidement refroidies dans l'eau glacée.

Les épreuves de comparaison sont faites dans du lait « reconstitué » à partir d'un lait écrémé sec dont l'aptitude à la coagulation est très régulière (Ets Lesaffre); nous employons une solution à $10 \mathrm{~g}$ pour $100 \mathrm{ml}$ de solution $0,003 \mathrm{M}$ de chlorure de calcium ; le $\mathrm{pH}$ final est exactement 6,5 .

\section{4) Séparation du phosphocaséinate}

Le lait est centrifugé durant $30 \mathrm{mn}$ à $0^{\circ} \mathrm{C}$, avec un champ maximum de $36000 \mathrm{~g}$ (ultracentrifugeuse Sorvall). Le sédiment recueilli au fond des tubes est redispersé dans le surnageant d'un autre lait.

\section{III. - RESULTATS}

\section{1) Coagulation dans le lait cru}

Avec tous les laits de vache crus examinés on a observé que la relation dite de Storch et Segelke (1874) n'est pas vérifiée avec l'enzyme fongique pour les temps de coagulation longs. Dans tous les cas, elle est vérifiée avec la présure animale, c'est-à-dire que la courbe reliant les temps de coagulation ( $t$ ) à la dilution (d) (inverse de la concentration) est une droite de 3 à $50 \mathrm{mn}$. La droite passe par l'origine et sa pente permet de mesurer l'activité de l'enzyme. Les figures 1 et 2 , avec ce mode de représentation, montrent que la partie droite donnée par la Fromase en lait cru ne correspond qu'au début de la courbe. Avec un même lait, les différents lots de Fromase donnent le même résultat. Avec le même enzyme, la longueur de la 
partie droite varie quelque peu d'un lait à l'autre de 8 à $15 \mathrm{mn}$; par exemple, un lait cru de mélange de la région de Toul donne une droite jusqu'à $10 \mathrm{mn}$ environ (fig. 1, courbe Fc) et un lait cru de mélange de la région de Nancy, jusqu'à $15 \mathrm{mn}$ environ (fig. 2, courbe Fc). Ce fait traduit les différences d'aptitude à la coagulation des deux laits considérés ; la partie droite correspond aux mêmes dilutions (de 5000 à 15000 ), mais les pentes ne sont pas les mêmes.

\section{2) Coagulation dans le lait chauffé}

Avec le lait reconstitué à partir de lait sec, tous les lots de Fromase ont donné une droite, pour des dilutions correspondant à des temps de coagulation compris entre 3 et $55 \mathrm{mn}$; par exemple, courbe Fr (fig. 1).

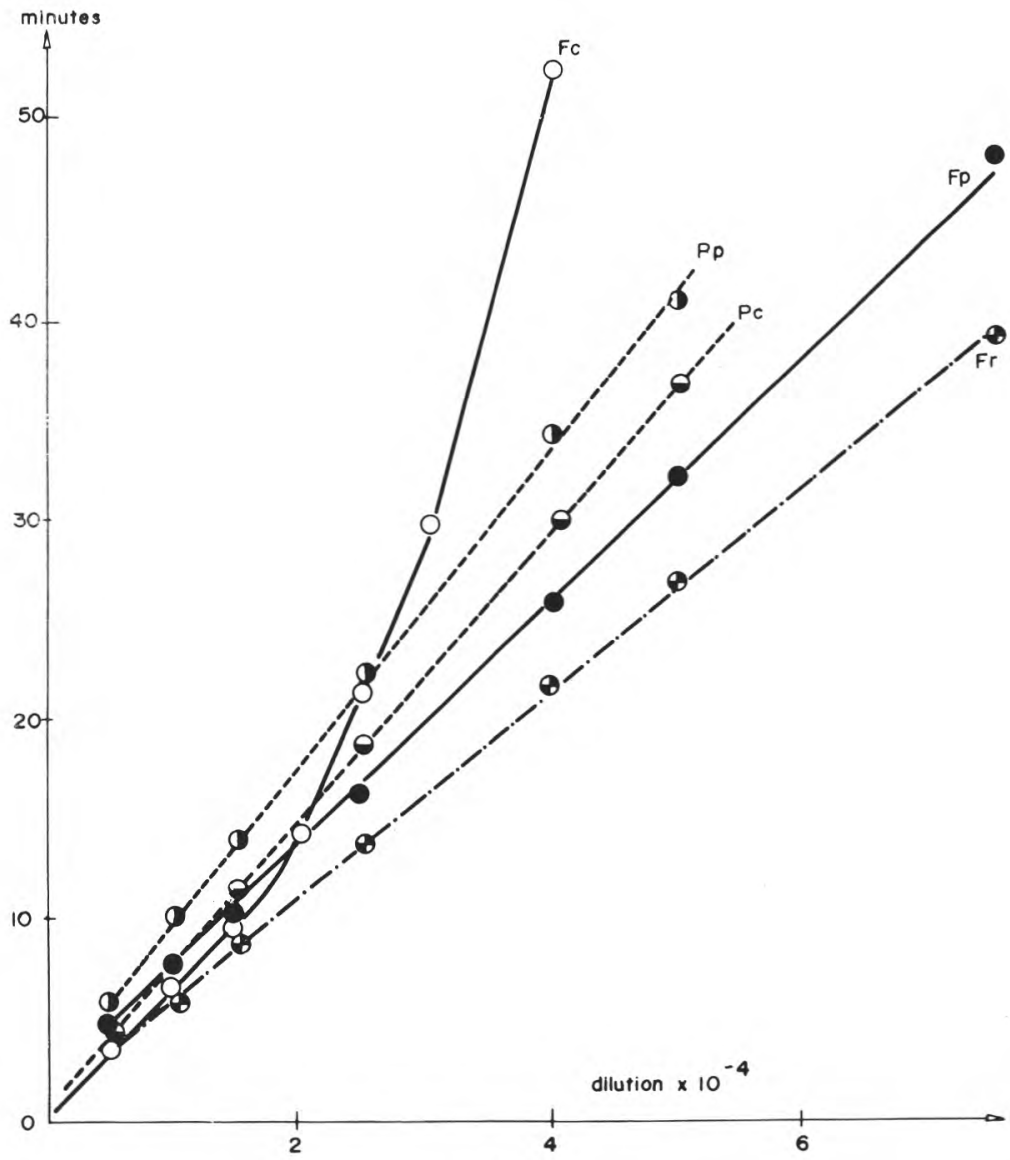

fig. 1

Evolution du temps de coagulation en fonction de la dilution de l'enzyme Fromase $(\mathrm{F})$ et de la présure $(\mathrm{P})$ à $32^{\circ} \mathrm{C}$, dans le lait cru $(\mathrm{c})$, pasteurisé à $72^{\circ} \mathrm{C} / 20 \mathrm{~s}(\mathrm{p})$ ou reconstitué $(\mathrm{r})$. 
Le chauffage du lait cru à $72^{\circ} \mathrm{C}$ pendant $20 \mathrm{~s}$ (pasteurisation HTST) rétablit l'aptitude normale à la coagulation (courbe Fp, fig. 1). La pente de la droite est presque toujours un peu inférieure à celle de la partie droite obtenue avec le lait $\mathrm{cru}$, ce qui traduit une légère amélioration de la coagulabilité. La présure animale donne des droites dans tous les cas, le chauffage augmentant les temps de coagulation (courbes $\mathrm{Pc}$ et $\mathrm{Pp}$, fig. 1).

Le chauffage du lait cru pendant le même temps (20 s) en dessous de $72^{\circ} \mathrm{C}$, a des effets variables selon la température atteinte. La " thermisation » à $60-65^{\circ} \mathrm{C}$ a peu d'effet ; on constate cependant

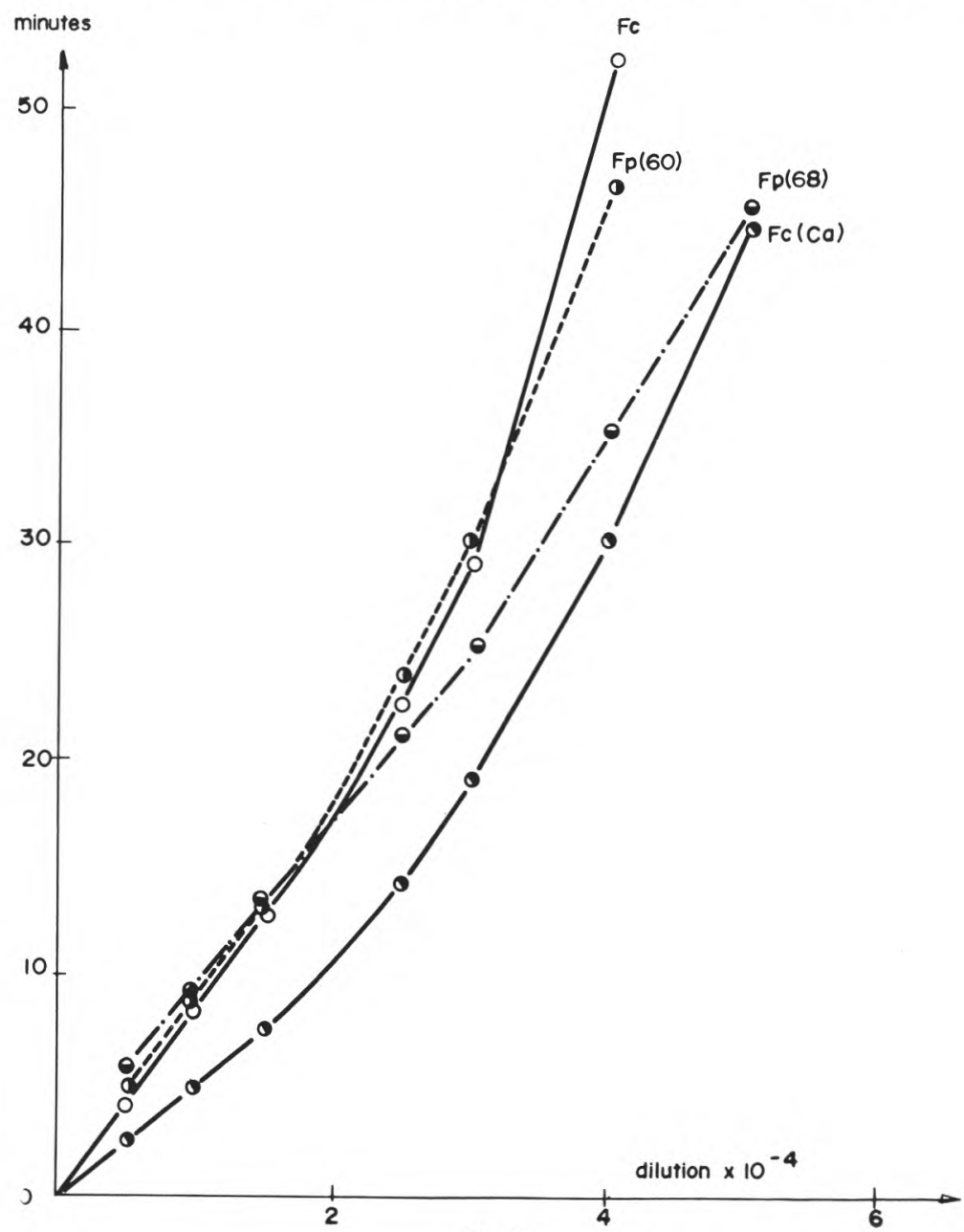

fig. 2

Evolution du temps de coagulation en fonction de la dilution de l'enzyme Fromase $(\mathrm{F})$ à $32^{\circ} \mathrm{C}$, dans le lait cru $(\mathrm{c})$, le lait additionné de $0,15 \mathrm{~g} / \mathrm{l} \mathrm{de}$ chlorure de calcium (Ca), dans le lait chauffé durant $20 \mathrm{~s}$ à $60^{\circ} \mathrm{C}(60)$ et à $68^{\circ} \mathrm{C}(68)$. 
que la partie droite de la courbe $t / d$ s'allonge. Le chauffage à $68^{\circ} \mathrm{C}$ réduit l'inhibition, qui n'apparaît que pour des dilutions plus grandes, correspondant à des temps de coagulation supérieurs à $25 \mathrm{mn}$ (fig. 2, courbe Fp-68).

Pour une durée de chauffage beaucoup plus longue, de $5 \mathrm{mn}$, la température pour laquelle la courbe redevient une droite est voisine de $60^{\circ} \mathrm{C}$.

\section{3) Effet de l'addition de calcium}

L'addition de chlorure de calcium au lait raccourcit les temps de coagulation, mais l'anomalie des courbes $t / d$ persiste (fig. 2, courbe Fc-Ca); on observe que la partie droite est plus limitée ; les temps de coagulation sont proportionnels jusqu'à $8 \mathrm{mn}$.

\section{4) Séparation du phosphocaséinate}

On a centrifugé un lait cru de ferme et le lait standard (reconstitué à partir de la poudre de lait) et l'on a séparé, d'une part, la caséine native sous forme de phosphocaséinate et, d'autre part, le surnageant qui contient les protéines solubles. Deux laits mixtes ont été obtenus en dispersant le sédiment d'un lait dans le surnageant de l'autre.

Msc : surnageant de lait cru + phosphocaséinate de lait standard.

$M p c$ : phosphocaséinate de lait cru + surnageant de lait standard.

La figure 3 montre que le lait mixte $M s c$, contenant les protéines solubles du lait cru, donne une courbe ne vérifiant pas la relation de Stork et Segelke (comme le lait cru C), alors que le lait mixte $M p c$, qui ne contient que le phosphocaséinate du lait cru, donne une droite (comme le lait standard). La différence entre C et Msc à la plus forte dilution, $\left(6 \times 10^{-4}\right)$ n'est pas significative, car dans une autre expérience l'écart des temps était inversé.

\section{IV. - DISCUSSION}

\section{1) Aspects scientifiques}

La forme des courbes du temps de coagulation en fonction de la dilution traduit probablement un phénomène d'inhibition de l'enzyme dans le lait cru, qui commence à se manifester lorsque la dilution atteint 20000 . Il s'agit de la dilution du produit commercial qui doit contenir environ 10 p. 100 d'enzyme pur ; la concentration en enzyme serait donc de $5 \mu \mathrm{g} / \mathrm{ml}$ de lait.

Il est probable que l'inhibition résulte de la combinaison stoechiométrique d'un inhibiteur avec l'enzyme. Si elle se faisait 


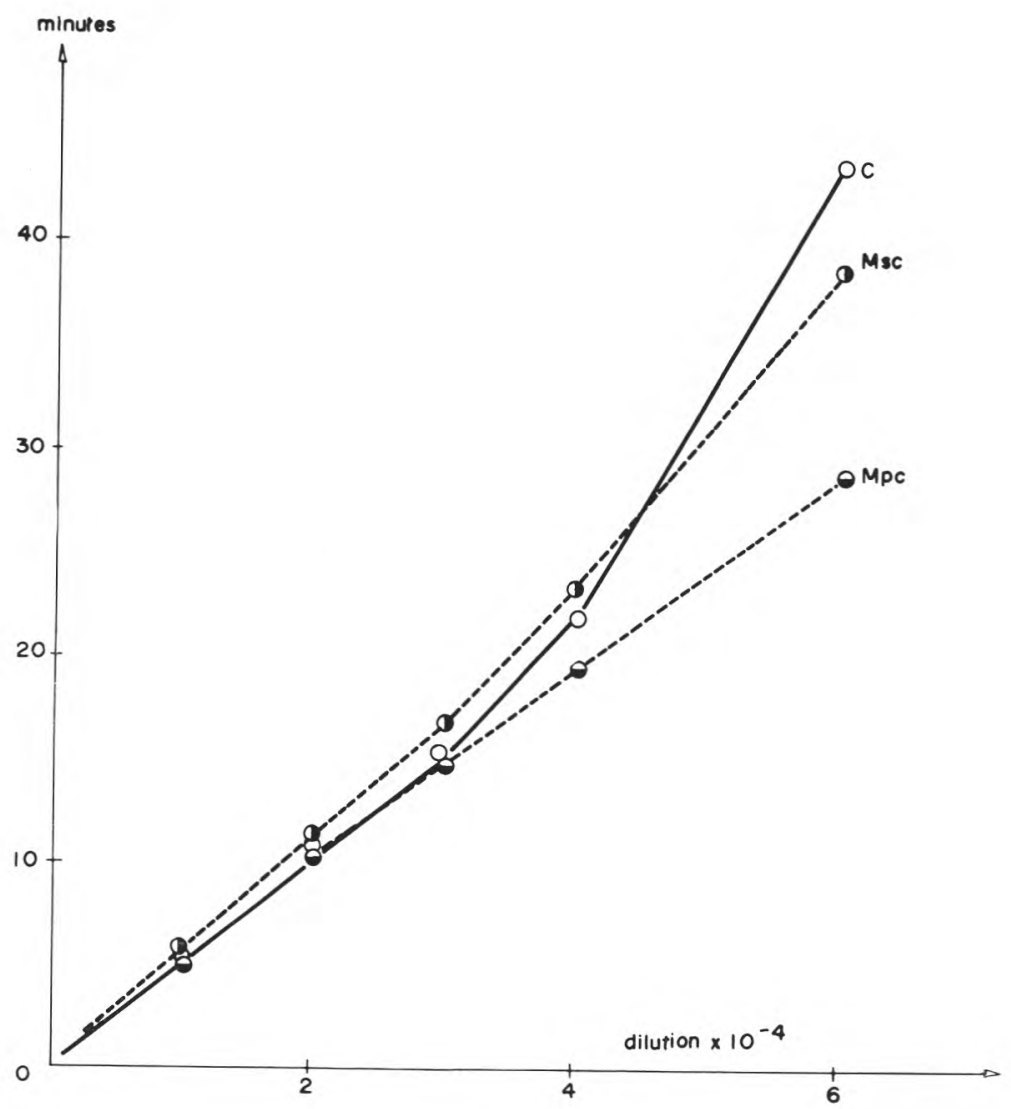

fig. 3

Temps de coagulation en fonction de la dilution de l'enzyme Fromase, à $32^{\circ} \mathrm{C}$, du lait $\mathrm{cru}(\mathrm{c})$, du lait mixte contenant le surnageant du lait cru (Msc) et du lait mixte contenant le phosphocaséinate du lait cru (Mpc).

molécule à molécule, comme on l'a suggéré dans le cas de l'ovomucoïde et de la trypsine, et si on prenait pour poids moléculaire de l'enzyme 38 000, (valeur donnée par Ottesen et Rickert (1970) pour une autre souche de Mucor miehei), on pourrait estimer la quantité d'inhibiteur à $1,3 \times 10^{-7}$ environ, car c'est approximativement la concentration molaire de l'enzyme pur à la dilution 20000 .

Le lait cru de vache ne contiendrait donc qu'une faible quantité de la substance inhibitrice. Celle-ci est sans doute de nature protéique ; elle est inactivée dans la zone de dénaturation des immuno-globulines du lait, fixée vers $70^{\circ} \mathrm{C}$ par Larson et Rolleri (1955) ; ces globulines 
font partie de la fraction des "protéines solubles ». Les expériences d'ultracentrifugation confirment que c'est dans cette fraction que se trouve l'inhibiteur, et non pas dans les micelles de caséine.

Il est peu probable que l'inhibition concerne la deuxième phase de la coagulation seulement ; celle-ci résulte d'une protéolyse limitée de la caséine dans laquelle l'existence d'une liaison sensible à la chymosine a été démontrée par Jollès et Alais (1968). On ne voit pas comment un inhibiteur empêcherait la réunion des micelles de caséine dans le cas de l'enzyme étudié et non pas dans le cas de la chymosine, alors que le mode d'action est semblable. La théorie du colloïde protecteur soutenue par Puhan (1967) n'est pas satisfaisante pour expliquer le phénomène observé. Si une globuline retardait la coagulation du fait d'une association avec les micelles, le lait serait "lent ", comme le disent les spécialistes ; il serait résistant à l'action de l'enzyme indépendamment de la concentration de celui-ci ; l'allongement du temps de coagulation ne serait pas apparent seulement en dessous d'un certain seuil du rapport enzyme/substrat.

Les enzymologistes raisonnent habituellement sur la vitesse de réaction et sur la concentration de l'enzyme ou du substrat. Les

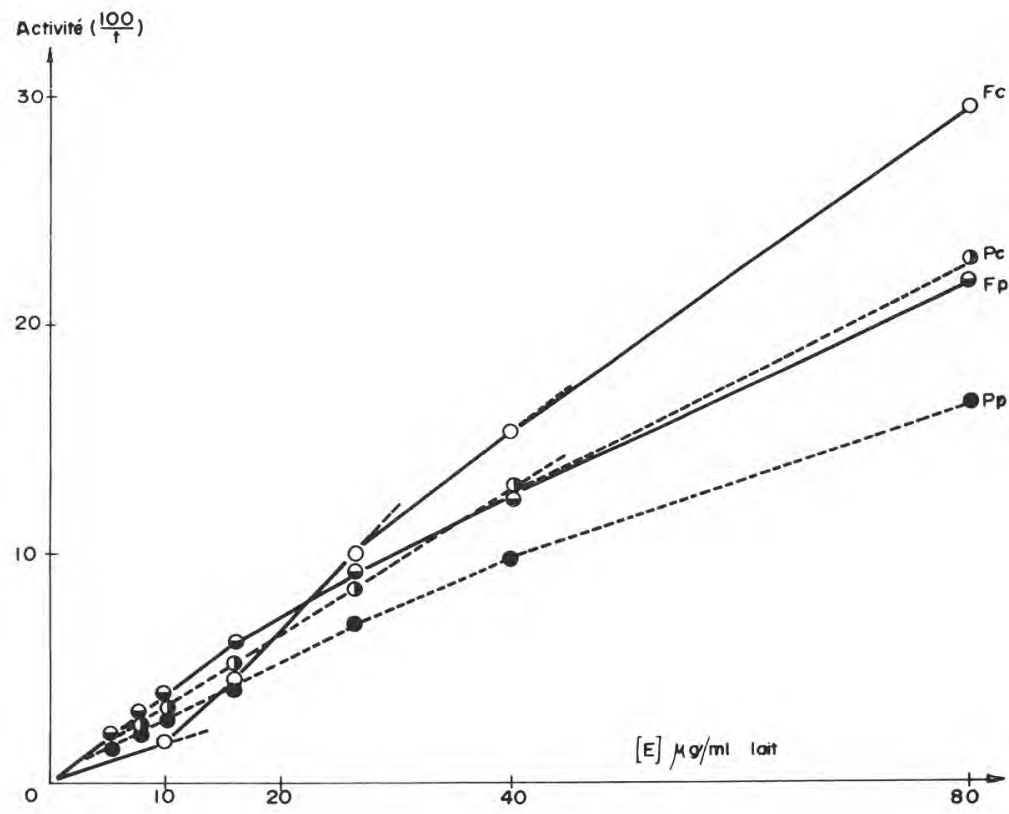

fig. 4

Activité coagulante de l'enzyme Fromase $(\mathrm{F})$ et de la présure $(\mathrm{P})$ en fonction de la concentration, à $32^{\circ} \mathrm{C}$, dans le lait cru (c) et le lait pasteurisé (p). 
courbes t/d employées d'ordinaire dans l'étude de la chymosine ne donnent donc pas une représentation adéquate. La représentation de la figure 4 reliant l'activité (inverse du temps de coagulation) à la concentration de l'enzyme permet une meilleure interprétation. L'inhibition apparaît à des concentrations en enzyme brut inférieures à $50 \mu \mathrm{g} / \mathrm{ml}$. En dessous de cette valeur, la vitesse de coagulation est plus faible que dans le lait chauffé, mais au-dessus, elle est plus grande. Lorsque l'inhibiteur est saturé par l'enzyme, on obtient une courbe de l'activité propre de l'enzyme dans le lait cru (Fc); on voit qu'elle est plus élevée que dans le lait pasteurisé (Fp) ce qui est normal; comme avec la présure, l'aptitude à la coagulation est diminuée après chauffage du lait.

Avec la présure, la courbe d'activité en fonction de la concentration en enzyme est une droite pour les concentrations moyennes et faibles. Lorsque la concentration croît, la vitesse de coagulation n'augmente pas proportionnellement. La même observation peut être faite avec l'enzyme Fromase, au-delà du seuil d'inhibition. La pente de la courbe va en diminuant dans les deux cas.

On sait depuis longtemps que la règle ancienne de Storch et Segelke (1874) n'est pas vérifiée dans toutes les conditions. Holter (1932) a montré qu'elle n'est valable que dans une zone de concentration en enzyme et de température assez limitée ; aux très faibles et très fortes concentrations, de même qu'à des températures au-dessus de $35^{\circ} \mathrm{C}$ ou au-dessous de $30^{\circ} \mathrm{C}$, le produit $\mathrm{c} \times \mathrm{t}$ (concentration $\times$ temps) n'est plus constant.

La vitesse de la "réaction primaire » (réaction enzymatique mesurée par la libération d'azote non-protéique) croît avec la concentration de l'enzyme, comme cela a été montré par Alais et al. (1953) et par Nitschmann et Bohren (1955), dans le cas de la présure. Mais il n'y a pas une concordance étroite entre l'achèvement de la réaction primaire et le point de coagulation, d'après les travaux de Foltmann (1959). Le temps qui sépare ces deux phénomènes ( time lag ») n'est pas totalement indépendant de la concentration en enzyme. Ceci rend difficile l'interprétation des courbes obtenues par la mesure du temps de coagulation, étant donné que l'activité n'est pas déterminée par la disparition du substrat ni par l'apparition d'un produit de la réaction.

Dans les travaux en cours, qui feront l'objet d'une prochaine publication, nous cherchons à identifier et à séparer les substances inhibitrices du lait cru et à connaître leur influence sur la réaction enzymatique proprement dite.

\section{2) Aspects technologiques}

L'inhibition de l'enzyme "Fromase » dans le lait cru se manifeste à des concentrations en enzyme qui couvrent une partie de la zone des durées de coagulation rencontrées en fromagerie. Dans les fabri- 
cations à coagulation rapide (moins de $15 \mathrm{mn}$ ) l'effet d'inhibition sera peu sensible ou inapparent. Dans les fabrications à durée de coagulation plus longue, il faudra en pratique un "surdosage " de l'enzyme, si l'on tient à conserver le temps de coagulation habituel en lait cru ou modérément thermisé, sans modifier la dose de chlorure de calcium et de levain lactique.

Dans de précédents essais en fromagerie de pâte cuite, nous avons observé, avec un autre enzyme coagulant fongique, un allongement du temps de coagulation de $12 \mathrm{mn}$ et un retard au tranchage de $15 \mathrm{mn}$, avec du lait thermisé à $63^{\circ} \mathrm{C}, 20 \mathrm{~s}$ (la dose d'enzyme avait été déterminée par l'épreuve de coagulation en lait reconstitué), (Ramet et Alais, 1973). Aucun problème ne se pose dans le cas de fabrication à partir de lait pasteurisé à $72^{\circ} \mathrm{C}$ et au-dessus.

L'emploi de nouveaux moyens de fabrication, qu'il s'agisse d'un procédé mécanique ou d'un agent biochimique, est motivé par des avantages technologiques ou économiques. Les avantages propres des enzymes coagulants fongiques sont maintenant bien connus ; mais il est inévitable qu'il y ait une contrepartie et il est bon d'en connaître les répercussions. L'inhibition exposée dans cette communication mérite d'être étudiée d'une part, pour son intérêt scientifique et, d'autre part, pour ses conséquences pratiques, même si celles-ci sont mineures et ne concernent pas la plus grande partie des fabrications fromagères qui utilisent le lait pasteurisé normalement.

\section{Remerciements}

Nous remercions Mlle Monique Grossi pour son efficace collaboration technique.

\section{Ré s u m é}

On a observé une inhibition de l'activité coagulante d'un enzyme produit par Mucor miehei (Fromase) dans le lait cru et dans le lait ayant subi un chauffage modéré. Le lait de vache contient, en faible proportion, une substance inhibitrice qui est inactivée vers $70^{\circ} \mathrm{C}$; elle se trouve dans les protéines solubles. En pratique, aucun problème ne se pose en fromagerie à partir du lait pasteurisé. Avec le lait cru ou modérément thermisé, un surdosage de l'enzyme est nécessaire si l'on ne veut pas modifier la dose de chlorure de calcium et de ferments lactiques.

\section{S u m m a r y}

The clotting activity of an enzyme derived from Mucor miehei (Fromase) is inhibited in raw milk and milk which has been moderately thermised. Cow milk contains, in small proportion, an inhibitory substance which is inactivated at about $70^{\circ} \mathrm{C}$; it is part of the 
soluble proteins. Pratically, no problem arises with pasteurized milk. With raw milk or with milk which has been moderately thermised, it is necessary to use a higher proportion of the enzyme if the calcium chloride and starters amounts cannot be altered.

Reçu pour publication le 13 mai 1974.

\section{Bibliographie}

Alais (C.), Mocouot (G.), Nitschmann (Hs.) et Zahler (P.) (1953). - Helv. Chim. Acta, 36, 1955-1968.

Briot (A.) (1900). - Thèse Sci., Paris, 22-46.

Foltmann (B.) (1959). - XV Internat. Dairy Congr., 2, 655-661.

Fossum (K.) (1970). - Acta path. microbiol. scand. Sektion B, 78, 350-362, 605-618, 741-754, 755-759.

Holter (H.) (1932). - Biochem. Z., 255, 160-170.

Jollès (J.), Alais (C.) et Jollès (P.) (1968). - Biochim. Biophys. Acta, 168, 591-593.

LaRson (B. L.) et Rolleri (G. D.) (1955). - J. Dairy Sci., 38, 351-355.

Laskowski (M.) Jr. et Laskowksi (M.) (1951). - J. Biol. Chem., 190, 563.

Laskowski (M.) Jr, Mars (P. H.) et Laskowski (M.) (1952). - J. Biol. Chem., 198, 745.

Nitschmann (Hs.) et Bohren (H. U.) (1955). - Helv. Chim. Acta, 38, 1953-1963.

Ottesen (M.) et Rickert (W.) (1970). - C.R. Lab. Carlsberg, 37, 301-325.

Porcher (C.) (1950). - Lait, 100, 1123 et (1931) Lait 101, 1.

Puhan (Z.) (1966). - Thèse Sci., Zürich.

Puhan (Z.) (1967). - Milchwissenschaft, 22, 133-136.

Ramet (J. P.) et Alais (C.) (1973). - Lait, 53, 154-162.

Singh (L. N.) et GANGULI (N. C.) (1973). - Milchwissenschaft, 28, 565-568.

Storch (V.) et SegelKe (Th.) (1974). - Milchzeitung, 3, 997.

Tsugo (T.) et Yamauchi (K.) (1953). - XIII Internat. Dairy Congr., 4, 641-644.

Vogel (R.), Trautshold (I.) et Werle (E.) (1968). - Natural proteinase inhibitors, Academic Press, New-York, 159. 